\title{
Influência da heterogeneidade ambiental sobre os atributos da comunidade de Chironomidae em lagoas de inundação neotropicais
}

\author{
Camila G. Bilia, Gisele D. Pinha, Danielle K. Petsch \& Alice M. Takeda
}

Programa de Pós-Graduação em Ecologia de Ambientes Aquáticos Continentais, Universidade Estadual de Maringá. Av. Colombo, 5790, 87020-900 Maringá, PR, Brasil. (cgentilinbilia@gmail.com)

\begin{abstract}
Influence of environmental heterogeneity on the attributes of Chironomidae community in neotropical floodplain lakes. The environmental structure is an important factor influencing the communities distribution. In this context, physically more complex environments sustain higher species richness than those simpler. We aimed to evaluate the influence of environmental heterogeneity on the atributes of Chironomidae community, such that to determe their spatial distribution in different lakes from the Upper Paraná River floodplain. Samples were collected quarterly from March to December 2011 in six environments (three lakes with connection and three lakes without connection to main river). We determined the lacustrine heterogeneity, by the Environmental Score of each lake, which was calculated by summing of chemical, physical and biological features of the lakes. The correlations between the environmental heterogeneity with the richness, density and diversity of chironomid species were significant. These results suggest that the lakes with the greatest environmental heterogeneity (or highest Environmental Score) are more able to support a higher number of individuals and taxa.
\end{abstract}

KEYWORDS. Richness, granulometric texture, distribution of organisms.

RESUMO. A estrutura do ambiente é um importante fator que influencia a distribuição das comunidades. Neste sentido, ambientes fisicamente mais complexos sustentam maior riqueza de espécies que aqueles mais simples. Este estudo teve por objetivo avaliar a influência da heterogeneidade ambiental sobre os atributos da comunidade de Chironomidae, determinando a distribuição espacial do grupo em diferentes lagoas da planície de inundação do alto rio Paraná. As coletas foram realizadas trimestralmente de março a dezembro de 2011, em seis ambientes (três lagoas com conexão e três lagoas sem conexão ao rio principal). A heterogeneidade das lagoas foi determinada pelo Escore Ambiental, o qual foi calculado através da soma das características físicas, químicas e biológicas das lagoas. As correlações entre o Escore Ambiental das lagoas com a riqueza, densidade e a diversidade de larvas de Chironomidae foram significativas. Tais resultados sugerem que as lagoas com maior Escore Ambiental ou maior heterogeneidade são mais propícias à suportar um número mais elevado de indivíduos e de táxons.

PALAVRAS-CHAVES. Riqueza, textura granulométrica, distribuição de organismos.

Heterogeneidade é definida como a complexidade e/ou variabilidade das propriedades de um sistema no espaço e/ou no tempo (Li \& Reynolds, 1995). Nesse sentido, a estrutura do ambiente é um importante fator que influencia a distribuição das comunidades (MACARTHUR \& MacArthur, 1961; Downes et al., 2000; Tolonen et al., 2001) e determina diferenças na diversidade, onde ambientes fisicamente mais complexos sustentam maior riqueza de espécies que aqueles mais simples (WiLLiams, 1963; Bell et al., 1991; Downes et al., 2000; Shostell \& Williams, 2007). Essa maior diversidade de espécies em relação à heterogeneidade ambiental está relacionada à maior disponibilidade de nichos e recursos alimentares, possibilitando assim a coexistência de um maior número de espécies em uma mesma área (SCOGNAmillo et al., 2003; TEws et al., 2004; Townsend et al., 2006).

Nesse sentido, a planície de inundação do alto rio Paraná é reconhecida por comportar uma grande heterogeneidade ambiental, compreendendo um mosaico de ambientes aquáticos (ThомAz et al., 2004), formado por lagoas com conexão, sem conexão, rios e canais secundários. Esses ambientes se diferenciam quanto à morfologia, hidrodinâmica e grau de comunicação com o rio principal, conferindo-lhes características limnológicas e físicas próprias (Тномаz et al., 1997), resultando em ambientes com diferentes níveis de heterogeneidade espacial.

Essa alta complexidade ecológica, produzida pelas interações entre a superfície da água, lençol freático e áreas ripárias (WARD \& TOCKNER, 2001), exerce um importante papel na manutenção desse ecossistema (SouzA FILHO \& Stevaux, 2004) e, consequentemente, na estrutura e dinâmica das comunidades aquáticas (HIGUTI \& TAKEDA, 2002; Train \& Rodrigues, 2004; TAKeda et al., 2004; SAntos \& Thomaz, 2005), que resultam em uma alta biodiversidade (WARD \& TOCKNER, 2001).

Dentre as comunidades aquáticas, Chironomidae representa um dos principais componentes presente nos sedimentos e na vegetação (Trivinho-Strixino \& Strixino, 1998; TAKEDA et al., 2004; LeNCIONI \& RosSARO, 2005). Esses organismos são amplamente distribuídos e, frequentemente, o grupo de insetos mais abundante nos ambientes aquáticos (Epler, 2001; Ferrington, 2008), colonizando uma grande variedade de biótopos, onde ocupam diferentes hábitats e vivem sob as mais diversas condições ambientais, destacando-se pelas elevadas densidade e diversidade (PINDER, 1986; PINDER, 1995; FERRINGTON, 2008).

Nas lagoas de inundação da planície do alto rio Paraná, as larvas de Chironomidae geralmente são 
os organismos bentônicos mais abundantes (HiguTI \& TAKEDA, 2002; TAKEDA et al., 2004) e são influenciadas tanto por processos biogeoquímicos quanto pela grande heterogeneidade de ambientes que podem determinar sua composição e distribuição (RossARO, 1991). Nesse contexto, testou-se a hipótese de que existe uma relação positiva entre a heterogeneidade ambiental e os atributos da comunidade de Chironomidae. Assim, espera-se que as lagoas mais heterogêneas comportem os maiores valores de riqueza, diversidade, densidade e equitabilidade de Chironomidae, $\mathrm{o}$ que determina diferenças na distribuição espacial do grupo na planície de inundação do alto rio Paraná.

\section{MATERIAL E MÉTODOS}

Área de estudo. O estudo foi desenvolvido em seis ambientes da planície de inundação do alto rio Paraná, englobando três lagoas com conexão e três lagoas sem conexão ao rio principal, pertencentes aos sistemas Ivinhema, Baía e Paraná (Fig. 1). As lagoas diferem quanto à conexão, profundidade e área (Tab. I).

Coleta de dados e procedimentos laboratoriais. As amostras foram coletadas em março, junho, setembro e dezembro de 2011, em seis lagoas com diferentes graus de conexão. A amostragem em cada lagoa foi realizada em um transecto, de uma margem a outra: margem direita, esquerda e centro. Em cada ponto dessas estações foram realizadas quatro amostragens de fundo, três para análise biológica e uma para análise sedimentológica e estimativa de teor de matéria orgânica, com draga tipo Petersen modificada $\left(0,0345 \mathrm{~m}^{2}\right)$. Após coletado, o material foi acondicionado em galões para ser lavado com o auxílio de um sistema de peneiras com malhas $2,0,1,0$ e $0,2 \mathrm{~mm}$. O material retido na peneira de $0,2 \mathrm{~mm}$ foi fixado com álcool $80 \%$ e triado sob microscópio estereoscópio.

As larvas de Chironomidae foram dissecadas e montadas em lâminas com solução de Hoyer, de acordo com metodologia proposta por TRIVINHO-STRIXINO \& StRIXINo (1995), e posteriormente identificadas ao menor nível taxonômico possível com o auxílio das chaves de identificação (Trivinho-Strixino \& Strixino, 1995; Trivinho-Strixino, 2011). As lâminas estão depositadas

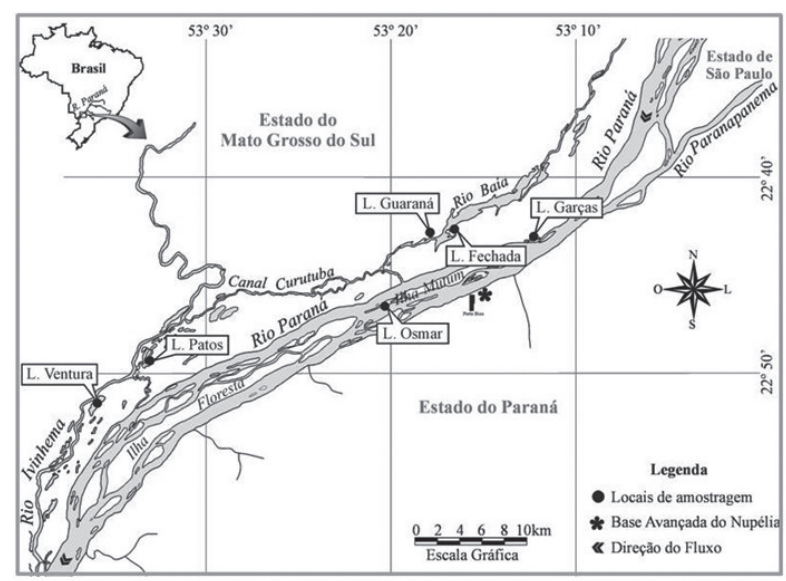

Fig. 1. Localização das estações de coleta, alto rio Paraná, Brasil. Lagoas com conexão: lagoa Patos, lagoa Guaraná e lagoa Garças. Lagoas sem conexão: lagoa Ventura, lagoa Fechada e lagoa Osmar.

na coleção do laboratório de Zoobentos da Universidade Estadual de Maringá (Nupelia).

A composição granulométrica foi determinada utilizando-se a escala de WeNTWORTH (1922). A estimativa do conteúdo de matéria orgânica do sedimento foi obtida pela queima da $20 \mathrm{~g}$ de sedimento seco em mufla a $560^{\circ} \mathrm{C}$, por cerca de quatro horas. Concomitante às coletas de fundo, os valores das variáveis abióticas da água foram obtidos pela equipe do laboratório de Limnologia Básica do Nupelia/UEM.

Análise de dados. Para a análise dos dados, nesse trabalho, optou-se pela estimativa média das variáveis, tanto abióticas, quanto bióticas de cada ambiente ao invés de transecto. Assim, para todas as análises, foram calculadas as médias entre as margens e o centro, resultando em um valor único para cada lagoa.

Para analisar as variações das características físicas e químicas da água nas estações e períodos amostrados, foi utilizada a Análise de Componentes Principais (GAUCH, 1986) para reduzir a dimensionalidade dos dados, facilitando assim, a interpretação dos resultados e a identificação de padrões espaciais entre as lagoas.

Os dados biológicos das larvas foram transformados em densidade média (ind $/ \mathrm{m}^{2}$ ) a partir da estimação pela área do amostrador.

Tab. I. Valores das variáveis morfométricas das lagoas amostradas, alto rio Paraná, Brasil (Prof., profundidade em metros; C., comprimento em metro; P., perímetro em metro; A., área em hectare). Adaptada de Souza FilHo et al., 2000.

\begin{tabular}{|c|c|c|c|c|c|c|c|}
\hline Sistema & Lagoa & Conexão & Localização & Prof. (m) & C. $(\mathrm{m})$ & P. (m) & A. (ha) \\
\hline \multirow{2}{*}{ Ivinhema } & L. Patos & Conectada & $\begin{array}{c}22^{\circ} 49^{\prime} 33,66^{\prime \prime} \mathrm{S} \\
53^{\circ} 33^{\prime} 9,9^{\prime \prime} \mathrm{W}\end{array}$ & 3,5 & 2065,5 & 14783,8 & 113,8 \\
\hline & L. Ventura & Isolada & $\begin{array}{l}22^{\circ} 51^{\prime} 23,7 ” \mathrm{~S} \\
53^{\circ} 36^{\prime} 1,02^{\prime \prime} \mathrm{W}\end{array}$ & 2,1 & 2984,82 & 4697,3 & 89,8 \\
\hline \multirow{2}{*}{ Baía } & L. Guaraná & Conectada & $\begin{array}{l}22^{\circ} 43^{\prime} 16,68^{\prime \prime} \mathrm{S} \\
53^{\circ} 18^{\prime} 9,24^{\prime \prime} \mathrm{W}\end{array}$ & 2,1 & 386,5 & 1058,3 & 4,2 \\
\hline & L. Fechada & Isolada & $\begin{array}{c}22^{\circ} 42^{\prime} 37,92^{\prime \prime} \mathrm{S} \\
53^{\circ} 16^{\prime} 33,06 ” \mathrm{~W}\end{array}$ & 2,46 & 624,4 & 1375,9 & 7,5 \\
\hline \multirow{2}{*}{ Paraná } & L. Garças & Conectada & $\begin{array}{l}22^{\circ} 43^{\prime} 27,18^{\prime \prime} \mathrm{S} \\
53^{\circ} 13^{\prime} 4,56^{\prime \prime} \mathrm{W}\end{array}$ & 2,0 & 2128,1 & 4338,2 & 14,1 \\
\hline & L. Osmar & Isolada & $\begin{array}{c}22^{\circ} 46^{\prime} 26,64^{\prime \prime} \mathrm{S} \\
53^{\circ} 19^{\prime} 56,16^{\prime \prime} \mathrm{W}\end{array}$ & 1,1 & 60,0 & 129,29 & 0,006 \\
\hline
\end{tabular}


Para a determinação da diversidade foi utilizado o Índice de Diversidade de Shannon-Wiener (H') (PIelou, 1975) e a equitabilidade (Pielou, 1966).

Através da composição e densidade das morfoespécies de Chironomidae, foi realizada uma Análise de Correspondência com Remoção do Efeito de Arco (DCA; GAUCH, 1986 - PC-Ord 5.0), que sumarizou os dados bióticos, reduzindo assim a dimensionalidade dos dados e facilitando a visualização de padrões espaciais.

Para avaliar a heterogeneidade das lagoas, utilizouse um Escore Ambiental, calculado através de pontuações, de acordo com as características heterogêneas das lagoas (Tab. II). Assim, consideramos alguns critérios que são mais relevantes para a comunidade de Chironomidae, como teor de matéria orgânica do substrato e a composição granulométrica, assim como as variáveis físicas e químicas da água, presença de macrófitas, etc. Cobertura arbórea sobre o corpo de água e composição vegetal do entorno foram trabalhadas como coisas distintas, pois algumas lagoas, mesmo apresentando densa vegetação nas margens, dada sua grande extensão, a presença da vegetação não chega a exercer uma cobertura vegetal efetiva sobre o corpo d'agua.

O Escore Ambiental final levou em conta todos os valores apresentados na Tabela II e foi calculado pelo somatório dos diferentes critérios analisados, apresentados na Tabela III, resultando em um valor adimensional, que indica o grau de heteregoneidade ambiental para cada lagoa.

Por meio do coeficiente de Correlação de Spearman ( $\rho)$, os dados de densidade das espécies de Chironomidae foram correlacionados com os dados abióticos (composição granulométrica, temperatura, condutividade, $\mathrm{pH}$ e oxigênio dissolvido). As associações serão inferidas com valores significativos para $\alpha=0,05$.

Todos os gráficos foram elaborados no software Statistica 7.0 (StaTSoft, 2005).

Tab. II. Variáveis físicas e químicas observadas no período de estudo entre as lagoas, alto rio Paraná, Brasil.

\begin{tabular}{|c|c|c|c|c|c|c|}
\hline & \multicolumn{6}{|c|}{ Lagoas } \\
\hline & Patos & Ventura & Guaraná & Fechada & Garças & Osmar \\
\hline Granulos e seixos (\%) & 0,04 & 0,55 & 0,00 & 0,00 & 0,00 & 0,00 \\
\hline Areia grossa (\%) & 0,04 & 0,55 & 0,00 & 0,00 & 0,00 & 0,00 \\
\hline Areia fina $(\%)$ & 23,59 & 28,31 & 19,14 & 20,92 & 13,35 & 9,69 \\
\hline Lama (\%) & 26,95 & 11,91 & 42,53 & 36,77 & 59,90 & 70,91 \\
\hline MOPG (\%) & 0,10 & 0,37 & 0,69 & 0,98 & 1,10 & 1,62 \\
\hline MOPF (\%) & 14,29 & 10,01 & 12,53 & 21,92 & 13,81 & 13,37 \\
\hline Temperatura $\left({ }^{\circ} \mathrm{C}\right)$ & 23,80 & 24,48 & 24,98 & 23,88 & 23,68 & 22,35 \\
\hline Oxigênio dissolvido (mg/l) & 4,63 & 5,95 & 6,78 & 5,60 & 6,45 & 3,15 \\
\hline Condutividade elétrica $(\mu \mathrm{S} / \mathrm{cm})$ & 39,00 & 33,78 & 52,58 & 28,88 & 27,10 & 46,90 \\
\hline $\mathrm{pH}$ & 6,50 & 6,78 & 6,88 & 6,20 & 6,38 & 6,18 \\
\hline Turbidez (NTU) & 8,43 & 74,13 & 6,23 & 12,45 & 39,85 & 7,75 \\
\hline Cobertura arbórea (\%) & 0,00 & 0,00 & 0,00 & 4,55 & 0,00 & 30,88 \\
\hline
\end{tabular}

\section{RESULTADOS}

Pelo critério de Kaiser-Guttman (autovalores maiores que 1), os dois primeiros eixos da PCA foram retidos para interpretação. Foram agrupados no eixo 1 as lagoas Garças e Osmar que pertencem ao rio Paraná, separando-as das lagoas Patos e Ventura que pertencem ao rio Ivinhema (Fig. 2). As lagoas do rio Ivinhema foram influenciadas positivamente pelos maiores valores de turbidez, areia fina e área, e as lagoas do rio Paraná foram agrupadas negativamente neste eixo, influenciadas pelos maiores valores de lama e matéria orgânica particulada grossa (Fig. 3).

Foram registradas 659 larvas de Chironomidae, identificadas em 47 morfoespécies de duas subfamílias (Chironominae e Tanypodinae), das quais Chironominae foi a mais abundante com 39 morfoespécies. A maior densidade de larvas foi encontrada na lagoa Osmar e a menor nas lagoas Guaraná e Fechada (Figs 4, 5). Chironomus fittkaui (Correia \& Strixino, 2007) foi a mais abundante na lagoa do Osmar e Procladius tipo B nas lagoas Patos e Ventura.

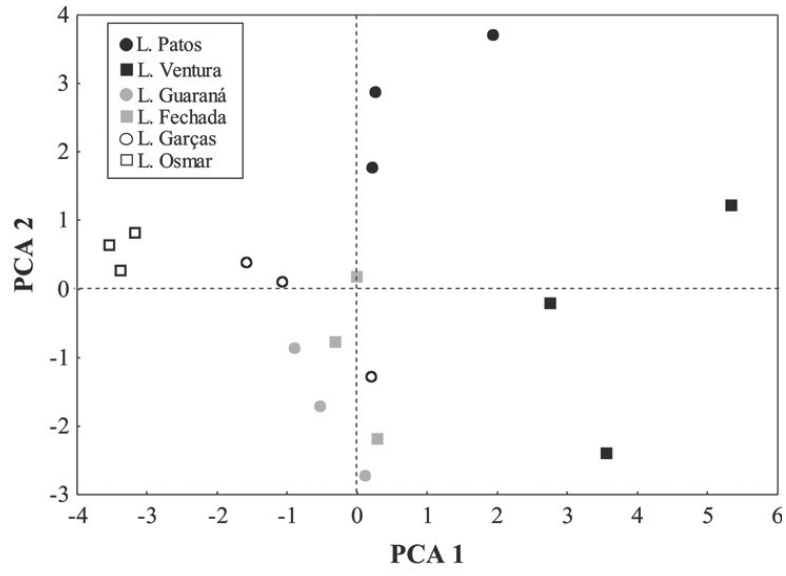

Fig. 2. Escores dos eixos 1 e 2 da Análise de Componentes Principais realizada com as variáveis físicas e químicas dos ambientes amostrados, alto rio Paraná, Brasil.

A maior diversidade e equitabilidade foram registradas nas lagoas Garças e Osmar. Nas lagoas Guaraná, Fechada e Patos foram obtidos os menores valores (Figs $5,6)$. Os maiores valores de riqueza foram verificados na 
Tab. III. Protocolo de campo para a determinação do Escore Ambiental.

\begin{tabular}{|c|c|c|}
\hline Critério & Avaliação & Nota \\
\hline \multirow{6}{*}{ Entorno lagoa } & Sem vegetação alguma (margem assoreada) & 0 \\
\hline & Vegetação composta por algum cultivar agrícola & 0,5 \\
\hline & Vegetação composta por pasto & 1,0 \\
\hline & Vegetação natural rasteira & 1,5 \\
\hline & Vegetação natural composta por gramíneas arbustos e arvoretas esparsos & 2,0 \\
\hline & Vegetação em diferentes estratos, com árvores, arbustos, e uma serapilheira bem formada & 2,5 \\
\hline \multirow{4}{*}{ Cor e cheiro da água } & Água de cor escura, visivelmente poluída, com forte odor podre & 0 \\
\hline & Água extremamente transparente, sem carga sólida alguma & 0,5 \\
\hline & Água escura, com uma concentração muito alta de compostos húmicos & 0,75 \\
\hline & Água de coloração intermediária, c/ compostos húmicos e outros tipos de material em suspensão & 1,0 \\
\hline \multirow{8}{*}{ Sedimento } & Porcentagem total de MO ultrapassa $70 \%$, material com forte odor podre & 0 \\
\hline & Porcentagem total de MOPF ultrapassa $20 \%$, alta porcent. Lama & 0,5 \\
\hline & Porcentagem total de lama ultrapassa $50 \%$ composta por argila compacta & 0,5 \\
\hline & Porcentagem de grânulos e seixos ultrapassa $50 \%$ com pelo menos 5\% MO & 2,0 \\
\hline & Porcentagens entre as partículas constituintes aproximadamente iguais & 1,5 \\
\hline & Alta porcentagem de areias com pouca MO e lama & 1,5 \\
\hline & Alta porcentagem de lama, composta por silte, alta porcentagem de areias mais finas & 1,5 \\
\hline & Alta \% (acima de 30\%) MOPG constituída de troncos, folhas e conchas & 2,0 \\
\hline \multirow{4}{*}{ Disponibilidade de margem } & Menor que $25 \%$ & 0,5 \\
\hline & Entre 25 e $50 \%$ & 1,0 \\
\hline & Entre 50 e $75 \%$ & 1,5 \\
\hline & Maior que $75 \%$ & 2,0 \\
\hline \multirow{3}{*}{ Fatores abióticos da água I } & Ausência de oxigênio no fundo/coluna de água & 0 \\
\hline & Ambiente com boa concentração de oxigênio (entre 2-4 mg/L) & 0,5 \\
\hline & Ambiente bem oxigenado até o sedimento (maior que $5 \mathrm{mg} / \mathrm{L}$ ) & 1,0 \\
\hline \multirow{2}{*}{ Fatores abióticos da água II } & Média da temperatura da água ao longo do ano maior que $30^{\circ} \mathrm{C}$ & 0 \\
\hline & Média da temperatura da água ao longo do ano entre $22-26^{\circ} \mathrm{C}$ & 1,0 \\
\hline \multirow{3}{*}{$\begin{array}{l}\text { Trofia e concentração de nutrientes } \\
\text { do ambiente }\end{array}$} & Altas concentrações de Fósforo e Clorofila caracterizando um ambiente eutrofizado & 0 \\
\hline & Concentrações muito baixas de nutrientes, caracterizando empobrecimento da água & 0,5 \\
\hline & Concentrações de nutrientes intermediária & 1,0 \\
\hline \multirow{4}{*}{$\begin{array}{l}\text { Cobertura arbórea sobre o corpo } \\
\text { aquático }\end{array}$} & Menor que $25 \%$ & 0,5 \\
\hline & Entre 25 e $50 \%$ & 1,0 \\
\hline & Entre 50 e $75 \%$ & 1,5 \\
\hline & Maior que $75 \%$ & 2,0 \\
\hline \multirow{4}{*}{ Macrófitas } & Macrófitas extremamente abundantes, cobrindo quase tota a área superfícial da lagoa & 0 \\
\hline & Macrófitas ou anfibias presentes nas margens, com dominância de poucas espécies & 0,5 \\
\hline & Macrófitas presentes nas margens, com presença de muitas espécies, sem dominância visual & 1,0 \\
\hline & Macrófitas submersas ou ausencia de macrofitas emersas ou bancos mto esparsos & 1,5 \\
\hline \multirow{4}{*}{ Influência do canal principal } & Grande influência de água do canal principal na lagoa (canal pequeno) & 0,5 \\
\hline & Lagoa muito isolada, distante em mais de 200 metros do canal principal & 0,5 \\
\hline & Influência do canal principal mínima (lagoa com canal) & 1,0 \\
\hline & Lagoa isolada (sem canal) & 1,0 \\
\hline
\end{tabular}

lagoa Osmar e Garças e os menores nas lagoas Guaraná, Fechada e Patos (Fig. 6).

Por meio da DCA, foi possível verificar que a lagoa Osmar se diferenciou das demais lagoas, comportando um número superior de espécies e uma composição característica, com maiores densidades de Chironomus tipo D, C. fittakaui, Ablabesmyia gr. annulata, Parachironomus cayapo (Spies et al., 1994) e Tanytarsus tipo D (Fig. 7).

A lagoa Osmar obteve o maior Escore Ambiental, seguido pela lagoa Garças, Ventura, Fechada, Guaraná e o valor mais baixo observado na lagoa Patos (Fig. 8). O Escore Ambiental correlacionou positivamente com todos os atributos analisados, mostrando tendências de aumento da densidade, do número de táxons e da diversidade de
Shannon-Wiener em relação ao aumento da heterogeneidade ambiental das lagoas, não sendo significativo apenas para a equitabilidade (Tab. IV).

\section{DISCUSSÃO}

A planície de inundação do alto rio Paraná comporta grande heterogeneidade de habitats aquáticos, o que promove alta diversidade biológica (AgostinHo et al., 2004; ThомAz et al., 2007). As diferentes lagoas de inundação apresentaram Escores Ambientais distintos, correlacionados positivamente com todos os atributos da comunidade. Estes resultados concordam com a nossa hipótese inicial de que quanto maior a heterogeneidade de um ambiente (avaliados 


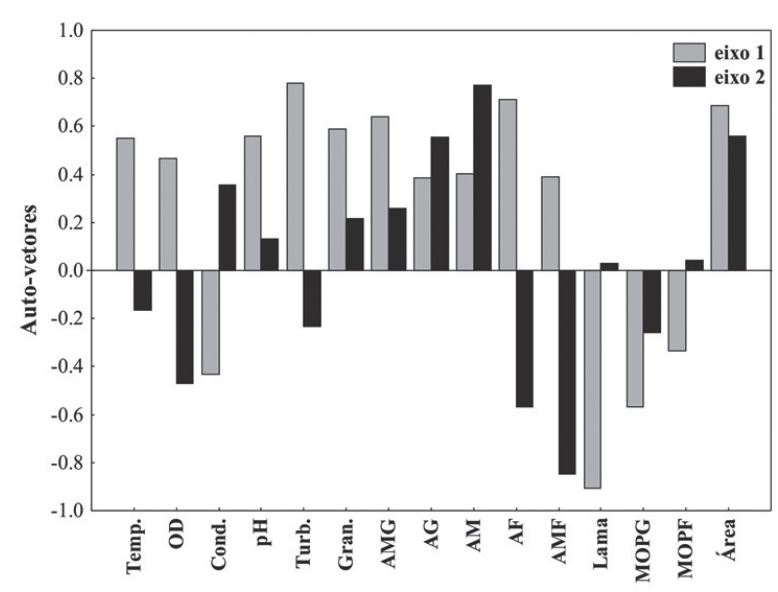

Fig. 3. Autovetores dos eixos 1 e 2 da Análise de Componentes Principais realizada com as variáveis físicas e químicas dos ambientes amostrados, alto rio Paraná, Brasil (AMG, areia muito grossa; AG, areia grossa; $\mathrm{AM}$, areia média; $\mathrm{AF}$, areia fina; $\mathrm{AMF}$, areia muito fina; $\mathrm{MOPG}$, matéria orgânica particulada grossa; MOPF, matéria orgânica particulada fina).

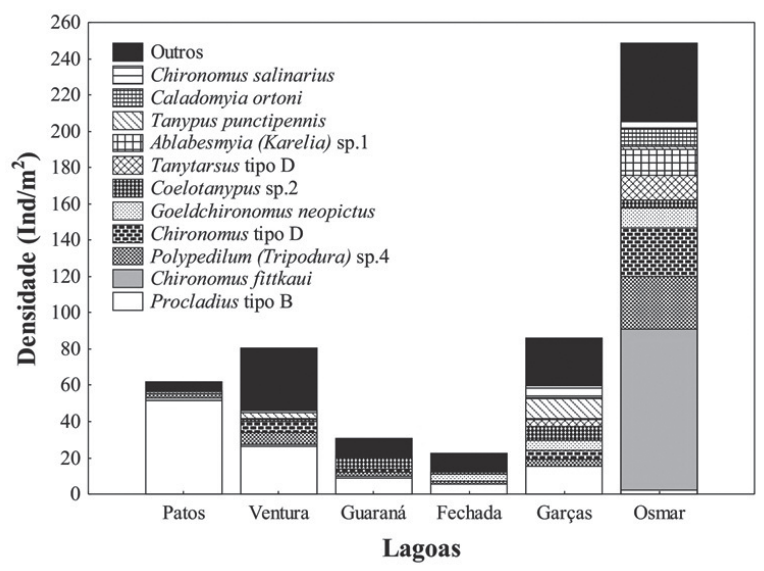

Fig. 4. Densidade (ind. $/ \mathrm{m}^{2}$ ) das larvas de Chironomidae nas seis lagoas amostradas na planície de inundação do alto rio Paraná, Brasil.

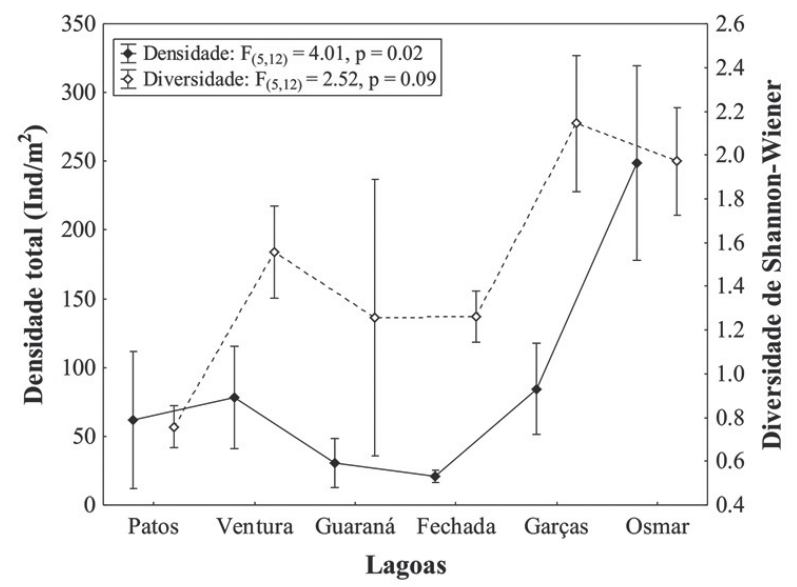

Fig. 5. Média \pm erro-padrão da densidade e diversidade das larvas de Chironomidae nas lagoas amostradas na planície de inundação do alto rio Paraná, Brasil. a partir do Escore Ambiental), apesar de termos verificado apenas uma tendência para a equitabilidade, maiores foram os valores dos atributos testados (densidade, diversidade, riqueza e equitabilidade) da comunidade.

A presença de vegetação arbórea densa verificada no entorno das lagoas Osmar e Garças possibilita aumento na entrada de galhos e folhas que passam a fazer parte do sedimento desses corpos de água. Dessa forma, estes ambientes tornam-se mais heterogêneos e fornecem abrigos e recursos alimentares para as larvas de Chironomidae (Sanseverino \& Neissimian, 2008). Segundo Callisto et al. (2002), a presença de vegetação ripária está associada com a riqueza dessa família e de outros invertebrados aquáticos e concordam com os resultados apresentados neste trabalho.

Em planícies de inundação, como a do alto rio Paraná, as variações no nível hidrométrico e a velocidade de correnteza dos rios principais alteram o tipo de substrato e a composição granulométrica do sedimento, fatores que influenciam diretamente os invertebrados bentônicos (HiguTI \& TAKEDA, 2002; Rosin \& TAKEDA, 2007; Rosin et al., 2010), que assim como a vegetação ripária, aumentam a heterogeneidade dos ambientes aquáticos.

O sedimento das lagoas pode sofrer grande influência dos rios principais, e o grau de influência varia de acordo com tipo da lagoa (com ou sem conexão com o rio principal), com a distância entre a lagoa e o rio principal e com a forma e tamanho do canal nas lagoas com conexão. De acordo com WARD et al. (1999), a conectividade é um dos fatores estruturantes principais das comunidades aquáticas e é fundamental para a manutenção da biodiversidade nos ecossistemas. Da mesma forma, a textura granulométrica e o teor de matéria orgânica são fatores determinantes que influenciam a distribuição e riqueza de quironomídeos (SARRIQUet et al., 2007; BRown, 2007), pois as larvas penetram no sedimento e a matéria orgânica proporciona recurso alimentar (ROBERTSON \& MILNER, 2001).

Apesar da semelhança na composição da textura granulométrica e teor de matéria orgânica entre as lagoas estudadas, as lagoas Osmar e Garças apresentaram alto teor de lama. Segundo Vos et al. (2002), lama e matéria orgânica são importantes para a alimentação e oferecem menos risco de predação quando comparados com sedimentos arenosos.

A relação entre área e perímetro demonstra que a disponibilidade de margem pode variar entre as lagoas. Nesse sentido, as lagoas podem apresentar grande área, porém pequeno perímetro, o que resulta em uma reduzida disponibilidade de margem. Por outro lado, uma lagoa com pequena área e grande perímetro gera uma grande disponibilidade de margem. Dessa forma, quanto maior a disponibilidade de margem, maior será a heterogeneidade da lagoa (Снiск \& MCIvor, 1994), portanto, maior será a disponibilidade de habitats e recursos alimentares para as larvas de Chironomidae e outros invertebrados (RAGONHA et al., 2013). Tais características podem ter contribuído para elevar a riqueza e densidade dos organimos na lagoa Osmar, o que a diferiu das demais pela análise de correspondência dendrítica. 


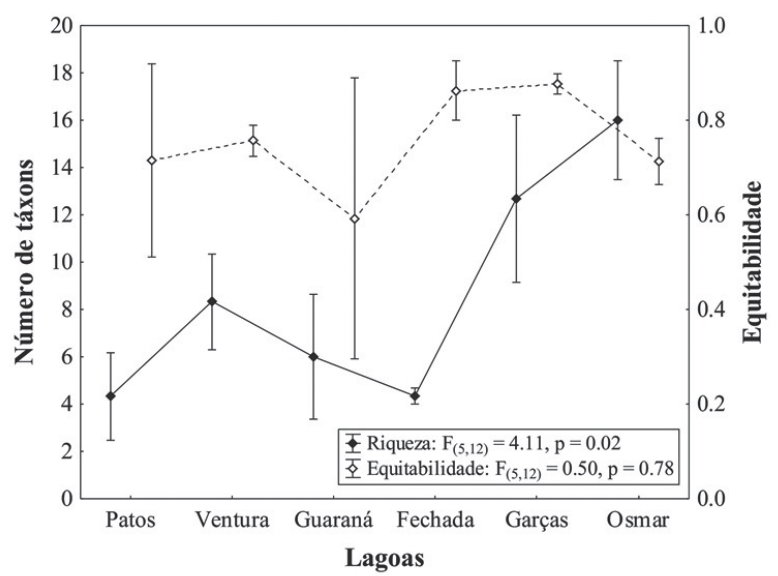

Fig. 6. Média \pm erro-padrão da riqueza e equitabilidade das larvas de Chironomidae nas lagoas amostradas na planície de inundação do alto rio Paraná, Brasil.

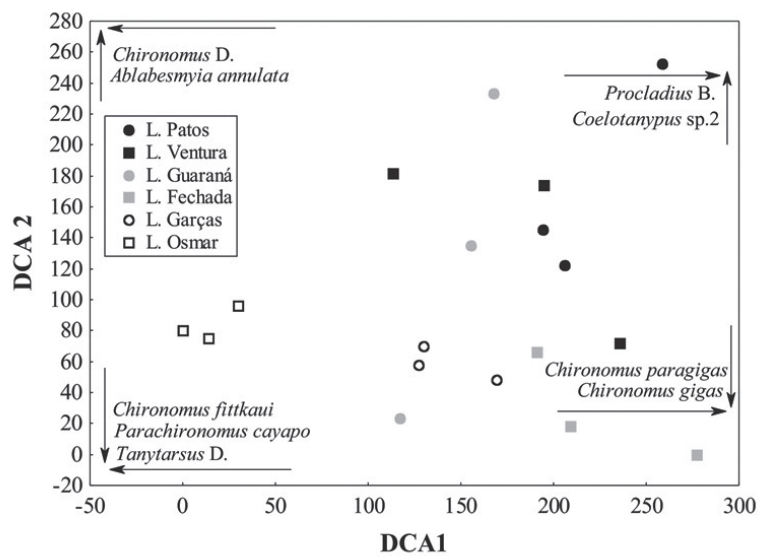

Fig. 7. Escores dos eixos 1 e 2 da Análise de Correspondência Dendrítica com remoção do efeito de arco realizada com as densidades de larvas de Chironomidae dos ambientes amostrados, alto rio Paraná, Brasil. As setas indicam as espécies mais positiva ou negativamente correlacionadas com cada eixo da análise.

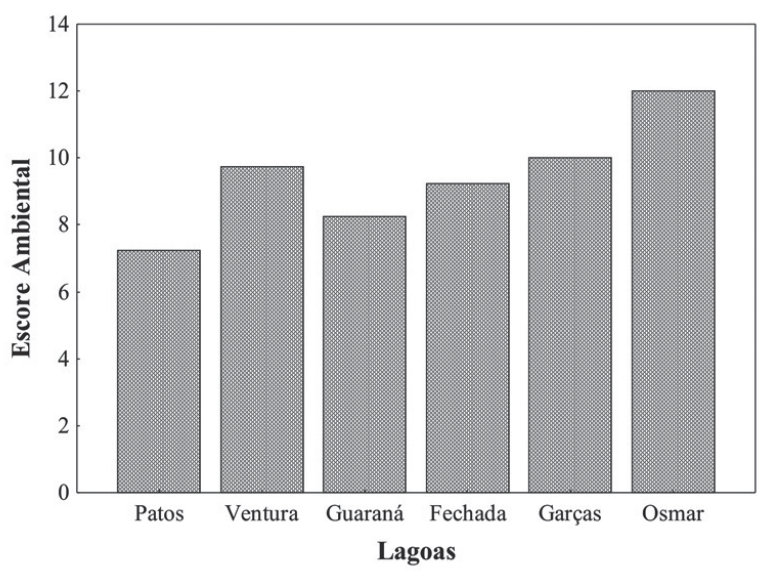

Fig. 8. Escore ambiental das seis lagoas amostradas na planície de inundação do alto rio Paraná, Brasil.
Tab. IV. Correlação de Spearman ( $\rho$ ) do Escore Ambiental das lagoas e os atributos (densidade, riqueza, equitabilidade e diversidade de ShannonWiener) das larvas de Chironomidae nas lagoas amostradas na planície de inundação do alto rio Paraná, Brasil, considerando a significância estatística da correlação para $\mathrm{p}<0,05$.

\begin{tabular}{lcc}
\hline & $\rho$ & $\mathrm{p}$ \\
\cline { 2 - 3 } Densidade (ind. $/ \mathrm{m}^{2}$ ) & 0,80 & 0,05 \\
Número de taxons & 0,88 & 0,02 \\
Equitabilidade & 0,25 & 0,62 \\
Diversidade (Shannon-Wiener) & 0,85 & 0,03 \\
\hline
\end{tabular}

Outro fator que influencia a heterogeneidade das lagoas são os bancos de macrófitas aquáticas. As lagoas da planície de inundação são predominantemente rasas e possuem comunidades extensas e diversificadas de macrófitas aquáticas. Estas plantas, quando em densidades moderadas, podem aumentar a heterogeneidade das zonas litorâneas, proporcionando hábitat e apoio para muitas comunidades aquáticas (JunK, 1970; WetZel, 1983). Nestas condições, os bancos de macrófitas exercem papel fundamental na ciclagem de nutrientes e na formação de detritos nos ambientes aquáticos (Petrucio \& Esteves, 2000; Binı et al., 2001) e servem ainda como local de oviposição dos adultos (WARD, 1992; TRIVINHO-STRIXINO \& Strixino, 1995) e abrigo para os primeiros estágios de muitas larvas de Chironomidae. Quando há macrófitas submersas, além das emersas, o ambiente torna-se mais heterogêneo, como verificado na lagoa Osmar.

Neste trabalho, os menores valores do Escore Ambiental foram atribuídos para as lagoas com densos bancos de macrófitas emergentes pelo fato de que em altas densidades, as macrófitas aumentam consideravelmente a entrada de matéria orgânica no sedimento, o que por sua vez, pode aumentar a demanda por oxigênio, reduzindo assim as concentrações deste localmente e afetando negativamente a comunidade de larvas. Além disso, densos bancos de macrófitas podem interferir com a chegada das larvas recém-eclodidas ao substrato, o que reduz a densidade de indivíduos do sedimento.

Todas essas características nas lagoas contribuem para o aumento da heterogeneidade ambiental, alterando a estrutura dos ambientes, que é um fator determinante na distribuição das comunidades (Downes et al., 2000; TOLONEN et al., 2001). Os ambientes mais complexos sustentam maior diversidade e riqueza de espécies que os ambientes mais simples (Williams, 1964; Bell et al., 1991; Downes et al., 2000; Shostell \& Williams, 2007), pois quanto maior a heterogeneidade ambiental, maior é a disponibilidade de nichos e de recursos alimentares (TEws et al., 2004; TownSEND et al., 2006).

No contexto de preservação de ecossistemas aquáticos, manter o entorno bem preservado, com a presença de densa vegetação ciliar, assim como evitar a entrada de poluentes orgânicos, que reduzem a qualidade da água e permitem a proliferação de algas e alguns tipos macrófitas, é essencial para alcançar maiores valores no 
Escore Ambiental. Como os valores do Escore Ambiental refletem diretamente na heterogeneidade ambiental, quanto maior a heterogeneidade de um ambiente, maior é o número de indivíduos e de táxons encontrados. Portanto, destaca-se a importância da manutenção de ambientes heterogêneos para a riqueza e distribuição das larvas de Chironomidae, assim como de outras comunidades aquáticas.

Agradecimentos. Os autores agradecem ao CNPq e a CAPES pelas bolsas de graduação e pós-graduação concedidas, respectivamente e ao programa PELD/CNPq pelo apoio financeiro; ao Nupélia pelo apoio logístico e ao Laboratório de Limnologia Básica do Nupélia/UEM, pelo fornecimento dos dados das variáveis da água.

\section{REFERÊNCIAS BIBLIOGRÁFICAS}

Agostinho, A. A., Thomaz, S. M. \& Gomes L. C. 2004. Threats for biodiversity in the floodplain of the Upper Paraná River: effects of hydrological regulation by dams. Ecohydrology and Hydrobiology 4:255-268

Bell, S. S.; Mccoy, E. D. \& Mushinsky, H. R. 1991. Habitat Structure: the Physical Arrangement of Objects in Space. London, New York, Chapman \& Hall. 438p.

BinI, L. M.; Thomaz, S. M. \& SouZa, D. C. 2001. Species richness and beta - diversity of aquatic macrophytes in the Upper Paraáa River foodplain. Archiv Fur Hydrobiologie 151(3):511-525.

BRown, B. L. 2007. Habitat heterogeneity and disturbance influence patterns of community temporal variability in a small temperate stream. Hydrobiologia 586:93-106.

Callisto, M.; Barbosa, F. A. R. \& Moreno, P. 2002. The influence of eucalyptus plantations on the macrofauna associated with Salvinia auriculata in southeast Brazil. Brazilian Journal of Biology 62(1):63-68

Снiск, J. H. \& McIvor, C. C. 1994. Patterns in the abundance and composition of fishes among beds of different macrophytes: viewing a littoral zone as a landscape. Canadian Journal of Fisheries and Aquatic Sciences 51:2873-2882.

Downes, B. J.; Lake, P. S.; Schreiber, E. S. G. \& Glaister, A. 2000. Habitat structure, resources and diversity: the separate effects of surface roughness and macroalgae on stream invertebrates. Oecologia 123:569-581.

EPLER, J. H. 2001. Identification Manual for the Larval Chironomidae (Diptera) of North and South Carolina. Crawfordwille, Special Publication. 526p.

Ferrington, L. C. 2008. Global diversity of non-biting midges (Chironomidae; Insecta-Diptera) in freshwater. Hidrobiologia 595(1):447-445.

GAUCH, J. R. 1986. Multivariate analysis in community ecology. Cambridge, Cambridge University Press. 298p.

Higuti, J. \& TAKedA, A. M. 2002. Spatial and temporal variation in of Chironomid larval (Diptera) in two lagoons and two tributaries of the Upper Paraná River floodplain, Brazil. Brazilian Journal of Biology 62(4):807-818.

JUNK, W. J. 1970. Investigations on the ecology and production-biology of the "floating meadows" Paspalum-Echinochloetum on the Middle Amazon. I. The floating vegetation and its ecology. Amazoniana 2(4):449-495.

Lencioni, V. \& Rossaro, B. 2005. Microdistribution of chironomids (Diptera: Chironomidae) in Alpine streams: an autoecological perspective. Hydrobiologia 533:61-76.

Li, H. \& Reynolds, J. F. 1995. On definition and quantification of heterogeneity. Oikos 73(2):280-284.

MacArthur, R. H. \& MacArthur, J. W. 1961. On bird species diversity. Ecology 42:594-598.

Petrucio, M. M. \& Esteves, F. A. 2000. Uptake of nitrogen and phosphorus in the water by Eichhornia crassipes and Salvinia auriculata. Revista Brasileira de Biologia 60(2):229-236.

Pielou, E. C. 1966. The measure ments of diversity in different types of biological colletions. Journal Theoretical Biology 13:131-144. . 1975. Ecological diversity. New York, Wiley. 165p.
Pinder, L. C. V. 1986. Biology of freshwater Chironomidae. Annual Review of Entomology 31:1-23.

1995. The habitats of chironomid larvae. In: Armitage, P. D.; Cranston, P. S. \& Pinder, L. C .V. eds. The Chironomidae. Biology and ecology of non-biting midges. London, Chapman \& Hall. 584p.

Ragonha, F. H.; Pinha, G. D.; Bilia, C. G.; Tramonte, R. P.; Silva, R. G. \& TAKEDA, A. M. 2013. The influence of shoreline availability on the density and richness of Chironomid larvae in Neotropical floodplain lakes. Bioikos 27(2):67-77.

Robertson, A. L. \& Milner, A. M. 2001. Coarse particulate organic matter: a habitat or food resource for the meiofaunal community of a recently formed stream? Archiv für Hydrobiologie 152:529-541.

Rosin, G. C.; Oliveira-Mangarotti, D. P. \& Takeda, A. M. 2010. Chironomidae (Diptera) community structure in two subsystems with different states of conservation in a floodplain of southern Brazil. Acta Limnologica Brasiliensia 22(3):276-286.

Rosin, G. C. \& TAKEDA, A. M. 2007. Larvas de Chironomidae (Diptera) da planície de inundação do alto rio Paraná: distribuição e composição em diferentes ambientes e períodos hidrológicos. Acta Scientiarum, Biological Sciences 29:57-63.

Rossaro, B. 1991. Chironomids of stony bottom streams: a detrended correspondence analysis. Hydrobiologia 122:79-93.

Sanseverino, A. M. \& Nessimian, J. L. 2008. Larvas de Chironomidae (Diptera) em depósitos de folhiço submerso em um riacho de primeira ordem da Mata Atlântica (Rio de Janeiro, Brasil). Revista Brasileira de Entomologia 52(1):95-104.

Santos, A. M. \& Thomaz, S. M. 2005. Diversidade de espécies de macrófitas aquáticas em lagoas de uma planície de inundação tropical: o papel de conectividade e do nível da água. Cadernos da Biodiversidade 5(1):16-24.

Sarriquet, P. E.; Bordenave, P. \& Marmonier, P. 2007. Effects of botton sediment restoration on interstitial habitat characteristics and benthic macroinvertebrate assemblages in a headwater stream. River Research and Applications 23:815-828.

Scognamillo, D.; Maxit, I. E.; Sunguist, M. \& Polisar, J. 2003. Coexistence of jaguar (Panthera onca) and puma (Puma concolor) in a mosaic landscape in the Venezuelan llanos. Journal of Zoology 259:269-279.

Shostell, J. M. \& Williams, B. S. 2007. Habitat complexity as a determinate of benthic macroinvertebrate community structure in cypress tree reservoirs. Hydrobiologia 575:389-399.

Souza Filho, E. E. \& Stevaux, J. C. 2004. Geology of the Paraná river valley in the vicinity of Porto Rico. In: Agostinho, A. A.; Gomes L. C. \& RoDRIGUES, L. eds. Structure and functioning of the Paraná river and its floodplain. LTER-site 6 (Peld-sitio 6). Maringá, EDUEM, p. 101-106.

Souza Filho, E. E.; Comunello, E.; Petry, A. C.; Russo, M. R.; Santos, A. M.; Rocha, R. R. A. \& LeIMIG, R. A. 2011. In: UnIVERSIDADE EsTADUAL DE MARIngÁ, Nupélia/PELD. ed. A planície de inundação do alto rio Paraná: site $6 \mathrm{PELD} / \mathrm{CENPq}$ - Relatório anual 2000. Maringá, UEM. Disponível em <http://www.peld.uem.br/Relat2000/2_2 CompBioticoDesLocAmost.PDF>. Acesso em 02.05. 2011.

StatSoft, Inc. (2005). Statistica (data analysis software system), version 7.1. Disponível em $<$ www.statsoft.com.

Takeda, A. M.; Fujta. D. S.; Komatsu, E.; Braga, P. C.; Oliveira, D. P.; Rosin, G. A.; Ibarra, J. A.; Silva, C. \& Anselmo, S. 2004. In: Agostinho, A. A.; Gomes, L. C. \& Rodrigues, L. eds. Influence of environmental heterogeneity and water level on distribution of zoobentos in the upper Paraná river floodplain (Baia and Paraná rivers). Structure and functioning of the Paraná river and its floodplain. LTER-site 6 (Peld-sitio 6). Maringá, EDUEM, p. 91-95.

Tews, J. U.; Brose, V.; Grimm, K.; Tielborger, M. C.; Wichmann, M.; Schwage, R. \& Jeltsch, F. 2004. Animal species diversity driven by habitat heterogeneity diversity: the importance of keystone structures. Journal of Biogeography 31:79-92.

Thomaz, S. M.; Roberto, M. C. \& Binı, L. M. 1997. Caracterização limnológica dos ambientes aquáticos e influência dos níveis fluviométricos. In: Vazzoler, A. E. A. M.; Agostinho, A. A. \& HAHN, N. S. A planície de inundação do alto rio Paraná: aspectos físicos, químicos, biológicos e sócioeconômicos. Maringá, EDUEM, p.73-102. 
Thomaz, S. M.; Pagioro, T. A.; Bini, L. M.; Roberto, M. C. \& Rocha, R. R. A. 2004. Limnology of the Upper Paraná Floodplain habitats: Patterns of Spatio-temporal Variations and Influence of the Water Levels. In: Agostinho, A. A.; Rodrigues, L.; Gomes, L. C.; Thomaz, S. M \& Miranda, L. E. eds. Structure and functioning of the Paraná river and its floodplain. LTER-site 6 (Peld-sitio 6). Maringá, EDUEM, p. 37-42.

Thomaz, S. M.; Bini, L. M. \& Bozelli, R. L. 2007. Floods increase similarity among aquatic habitats in river-floodplains systems. Hydrobiologia 579(1):1-13.

Tolonen, K. T.; Hamalainen, H.; Holopainen, I. J. \& Karjalainen, J. 2001. Influence of habitat type and environment variables on littoral macroinvertebrate communities in a large lake system. Archiv für Hydrobiologie 152:39-67.

Townsend, C. R.; Begon, M. \& Harper, J. L. 2006. Fundamentos em Ecologia. Porto Alegre, Artmed. 592p.

Train, S. \& Rodrigues, L. C. 2004. Phytoplankton assemblages. In: Thomaz, S. M.; Agostinho, A. A. \& Hahn, N. S. eds. The upper Paraná river and its floodplain: physical aspects, ecology and conservation. Leiden, Backhuys Publishers, p. 125-143.

Trivinho-Strixino, S. \& Strixino G. 1998. Goeldichironomus neopictus, a new species from the Southeast of Brazil: description and bionomic information (Insecta, Diptera, Chironomidae). Spixiana 21:271-278.

Trivinho-Strixino, S. 2011. Larvas de Chironomidae: Guia de identificação. São Carlos, UFSCar. Departamento de Hidrobiologia. Laboratório de Entomologia Aquática. Disponível em: $<$ http://sites. google.com/site/brazilianchironomids/keys2/trivinhostrixino2011>. Acesso em 05.2011.
Trivinho-Strixino, S. \& Strixino, G. B. M. A. 1995. Larvas de Chironomidae (Diptera) do Estado de São Paulo: Guia de Identificação e Diagnose dos Gêneros. São Carlos, PPG-ERN/ UFSCar. 229p.

Vos, J. H.; Teunissen, M.; Postma, J. F. \& Van Den Ende, F. P. 2002. Particle size effect on preferential settlement and growth rate of detritivorous chironomid larvae as influenced by food level. Stuttgart, Archiv für Hydrobiologie154:103-119.

Ward, J. V. 1992. Aquatic Insect Ecology. New York, Jonh Wiley \& Sons. $424 p$

WARD, J. V. \& TOCKNER, K. 2001. Biodiversity: toward a unifying theme for river ecology. Freshwater Biology 46(6):807-819.

WARD, J. V.; TOCKNER, K. \& SCHIEMER, F. 1999. Biodiversity of floodplain river ecosystems: ecotones and connectivity. Regulated Rivers 15:125-139.

Wentworth, C. K. 1992. A escale of grade and class terms for clastic sediments. Journal Geology 30:377-392.

Wetzel, R. G. 1983. Recomendation for future research on periphyton. In: Wetzel, R. G. ed. Periphyton of freshwater ecosystems. Dordrecht, Dr. W. Junk Publishers, p. 339-346.

Williams, C. B. 1963. Patterns in the Balance of Nature. New York, Academic Press. 324p. 